\title{
ADULT syndrome
}

INSERM

\section{Source}

INSERM. (1999). Orphanet: an online rare disease and orphan drug data base. ADULT syndrome. ORPHA:978

ADULT (Acro-dermo-ungual-lacrimal-tooth) syndrome is a rare ectodermal dysplasia syndrome (see this term) characterized by ectrodactyly, syndactyly, mammary hypoplasia, and excessive freckling as well as other typical ectodermal defects such as hypodontia, lacrimal duct anomalies, hypotrichosis, and onychodysplasia. 